\title{
Numerical Simulation of the Fatigue Behaviour of a Friction Stirred Channel Aluminium Alloy
}

\author{
Catarina Vidal $^{1}$, Ricardo Baptista ${ }^{2}$, and Virgínia Infante ${ }^{3, *}$ \\ ${ }^{1}$ Instituto de Plasmas e Fusão Nuclear, Instituto Superior Técnico, Universidade de Lisboa, Av. Rovisco Pais, 1049-001 Lisboa, Portugal \\ ${ }^{2}$ ESTSetúbal, Instituto Politécnico de Setúbal, Campus do IPS, Estefanilha, 2914-508 Setúbal, Portugal \\ ${ }^{3}$ LAETA, IDMEC, Instituto Superior Técnico, Universidade de Lisboa, Av. Rovisco Pais, 1049-001 Lisboa, Portugal
}

\begin{abstract}
Friction stir channelling (FSC) is one of the most promising innovations based on the friction stir concepts. FSC uses a non-consumable tool, similar to that used to perform the friction stir welding (FSW) process, to produce continuous and integral channels, with any path, in monolithic aluminium alloys components in a single step. This paper presents the mechanical behaviour of friction stirred channel components in particular the fatigue resistance of the channels analysed. Finite element analyses were performed taking into account the maximum stress values applied in experimental fatigue tests of the aluminium components. The energy release rate for different crack lengths was obtained using the software ABAQUS. The fatigue crack growth curve was established according to the Paris Law, and the crack propagation direction was calculated using the maximum tangential stress (MTS) criterion. During the analysis it was observed that the critical areas are located in the vicinity of the channels corners, as it was observed in the fracture surfaces of the experimental fatigue test results.
\end{abstract}

\section{Introduction}

The disruptive nature of the friction stir welding (FSW) process has led to many developments from the research and technological development institutions and industry. The FSW technology has been subjected to the most demanding quality standard requirements and used in challenging industrial applications over a wide range of structural and non-structural components, mainly in aluminium alloys. The disruptive character of the FSW process is emphasized by the numerous technic-scientific papers and patents published and the several friction stir based technologies that are being invented and developed since the original patent has been issued [1-4]. The friction stir channelling (FSC) technology, proposed for the first time in 2005 by Mishra as a method of manufacturing heat exchanging devices [5], is one of the most promising innovations based on the friction stir concepts. FSC uses a non-consumable tool, similar to that used to perform the FSW process, to produce continuous and integral channels, with any path, in monolithic aluminium alloys components in a single step.

The FSC technology involves complex interactions among a multiplicity of simultaneous thermo-mechanical processes. These interactions affect the heating and cooling rates, the plastic deformation and flow, the dynamic recrystallization phenomena and the mechanical integrity of the channel.

A typical FS channel cross-section macrograph is presented in Figure 1.

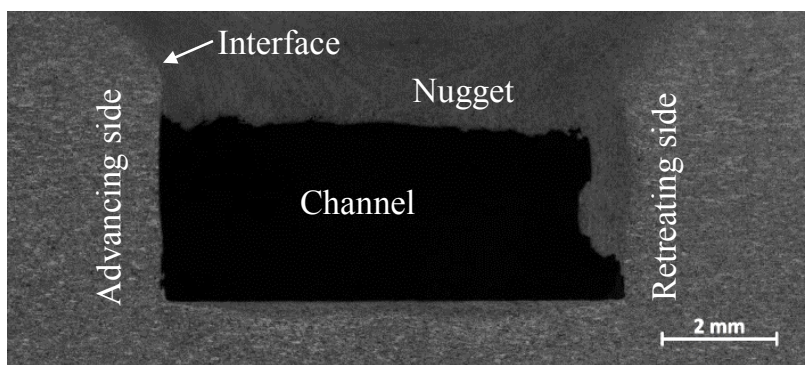

Figure 1. Cross-section macrograph of a FS channel.

The fatigue process can in general be divided into two main steps [6]: initiation of micro-cracks due to local accumulation of dislocations, high stresses at local points, plastic deformation around inhomogeneous inclusions or other imperfections; crack propagation, which causes permanent damage. The position and mode of fatigue crack initiation depends on the microstructure of the material, the type of the applied stress and geometry of the specimen [7]. The crack initiation periods can be very different and cracks can be initiated either on or under surface. Fatigue cracks initiation can be observed in stress concentration points where the

Corresponding author: virginia.infante $@$,tecnico.ulisboa.pt 
resistance area is smaller. The number of cycles, $N_{f}$ required for fracture can be determined from the number of stress cycles, $N_{i}$, required for the appearance of the initial crack in the material and the number of stress cycles, $N_{p}$ required for a crack to propagate from the initial to the critical crack length, when the final failure can be expected to occur $\left(N_{f}=N_{i}+N_{p}\right)$.

The interface between two different materials, is considered to show a weak behaviour, when compared with the base material or the added material. Whether one is considering a crack between a base material and a coating [8], a crack between to dissimilar material joined by friction stir welding [9] or a crack between two non-metallic materials [10], crack propagation rate on the interface will always be higher. This is attributed by Shah et al. [11] to the mismatch in the Young's modulus between the different materials. Therefore, studying the crack propagation along material interfaces is a complex mater and requires the use of different fracture parameters, as the energy release-rate, when considering crack propagation between to different elastic layers [11] or friction stir welds [9].

When a crack propagates along an interface between two different materials, the crack always behaves in mixed mode. Even in a pure mode I problem, the mismatch between the two materials Young's modulus, disrupts the symmetry of the problem. The crack tip is then always subjected to normal and tangential stresses. Therefore, crack propagation will occur in Mode I and Mode II, but these modes cannot be uncoupled. In other words, crack propagation will always be depended on the crack length and the materials properties mismatch. Unlike linear homogeneous conditions, the stress field near the crack tip does not show an inverse square root singularity. The stresses near the interface crack tip show an oscillatory behaviour (eq. 1), characterized by an oscillatory index (eq. 2), which is a function of the two materials properties.

$$
\begin{aligned}
& \sigma \sim r^{-\frac{1}{2}+\epsilon} \\
& \epsilon=\frac{1}{2 \pi} \ln \left(\frac{1-\beta}{1+\beta}\right)
\end{aligned}
$$

Where parameters $\beta$ and $\alpha$ are interface parameters defined by Dundurs [12]. The elastic mismatch parameter $\beta$, is a function of the Poisson's ratio and the material shear modulus (eq. 3). While parameter $\alpha$ measures the relative materials stiffness (eq. 4).

$$
\begin{aligned}
& \beta=\frac{\mu_{1}\left(1-2 \vartheta_{2}\right)-\mu_{2}\left(1-2 \vartheta_{1}\right)}{2\left(\mu_{1}\left(1-\vartheta_{2}\right)+\mu_{2}\left(1-\vartheta_{1}\right)\right)} \\
& \alpha=\frac{E_{1}-E_{2}}{E_{1}+E_{2}}
\end{aligned}
$$

The behaviour of the stress field along the crack interface, was defined by Rice [13]. The oscillatory behaviour of the stress singularity, can be define by eq. 5 , where the stress intensity factors for mode I and mode II, cannot be decoupled. $\sigma_{y y}+i \sigma_{y y}=\frac{K_{I}+i K_{I I}}{\sqrt{2 \pi r}} r^{i \epsilon}$

Crack propagation on materials interface is therefore more complex to analyse, as it requires the know the solution for the previous coupled stress intensity factors. Kakiuchi et al. [9] and Xuan et al. [14] have successfully used the energy release rate, $G$, to study interface crack propagation. This fracture parameter can be easily calculated by finite element method (FEM) and is more suitable for this type of problem. Considering the energy release rate for crack propagation, one can use the Paris Law in the following form:

$\frac{d a}{d N}=A \Delta G^{n}$

Where $\mathrm{A}$ and $\mathrm{n}$ are material parameters.

In this work finite element analyses were performed to determine the energy release rate for a given crack length. Considering the alternative version of the Paris Law in eq. 6, the elapsed number of fatigue cycles for a given crack increment can be calculate. This iterative process was used to predict fatigue crack propagation behaviour on the materials interface.

\section{Material and experimental details}

\subsection{Material}

The strain hardened aluminium alloy AA5083 in the H111 condition was used in this investigation. Its chemical composition and main mechanical properties obtained at room temperature are presented, respectively, in Tables 1 and 2.

Table 1. Chemical composition of AA5083-H111 [15].

\begin{tabular}{|l|l|l|l|l|l|}
\hline Element & $\mathrm{Al}$ & $\mathrm{Mg}$ & $\mathrm{Mn}$ & $\mathrm{Fe}$ & $\mathrm{Cr}$ \\
\hline Weight (\%) & 93.38 & 5.26 & 1.02 & 0.19 & 0.15 \\
\hline
\end{tabular}

Table 2. Mechanical properties of AA5083-H111 at room temperature [15].

\begin{tabular}{|l|l|}
\hline Vickers hardness & $92 \mathrm{HV} 0.5$ \\
\hline Yield strength & $210 \mathrm{MPa}$ \\
\hline Ultimate tensile strength & $375 \mathrm{MPa}$ \\
\hline Elongation & $18.25 \%$ \\
\hline Young's modulus & $68.9 \mathrm{GPa}$ \\
\hline
\end{tabular}

\subsection{Friction stirred channel production}

The friction stirred channel was produced on $15 \mathrm{~mm}$-thick rolled plate along the rolling direction using an ESAB LEGIO ${ }^{\mathrm{TM}}$ 3UL numeric control equipment. Plunge and dwell periods (zero tool travel speed) were performed under vertical position control and processing period (non-zero tool travel speed) was carried out under vertical force control.

In order to obtain a plate with an internal channel, a selected set of FSC parameters was implemented as shown in Table 3. A modular H13 steel tool that enables 
internal forced refrigeration was used. This tool is based on three main components: body, shoulder and probe. All tool's arrangement used had a cylindrical probe with $8 \mathrm{~mm}$ diameter and a $19 \mathrm{~mm}$ diameter plane shoulder with one spiral. The cylindrical probe used had left handed threads along its length, with a trapezoidal profile, a thread pitch of $3 \mathrm{~mm}$ and a depth of cut of $0.7 \mathrm{~mm}$. The geometric features of the shoulder used are presented in Table 4 . The tool was rotated in the counter clockwise direction. The tool tilt angle was $0^{\circ}$ for all the runs.

Table 3. FSC parameters.

\begin{tabular}{|l|l|l|l|}
\hline $\begin{array}{l}\text { Probe } \\
\text { length } \\
(\mathrm{mm})\end{array}$ & $\begin{array}{l}\text { Tool rotation } \\
\text { speed } \\
(\mathrm{rpm})\end{array}$ & $\begin{array}{l}\text { Tool travel } \\
\text { speed } \\
(\mathrm{mm} / \mathrm{min} .)\end{array}$ & $\begin{array}{l}\text { Vertical } \\
\text { force } \\
(\mathrm{kN})\end{array}$ \\
\hline 8 & 1100 & 50 & 1.5 \\
\hline
\end{tabular}

Table 4. Geometric features of the tool shoulder.

\begin{tabular}{|l|l|}
\hline Spiral pitch & Spiral height $(\mathrm{mm})$ \\
\hline 1 & 1 \\
\hline
\end{tabular}

In order to model the real geometry of the channel for the numerical simulations, its geometric parameters were measured (Figure 3).

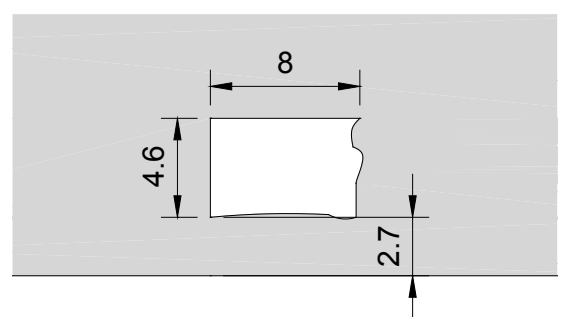

Figure 3. Geometry and dimensions of the channel produced by FSC.

\subsection{Fatigue testing}

Specimens for fatigue testing were produced according to the standard E 290-97a requirements as shown in Figure 4.
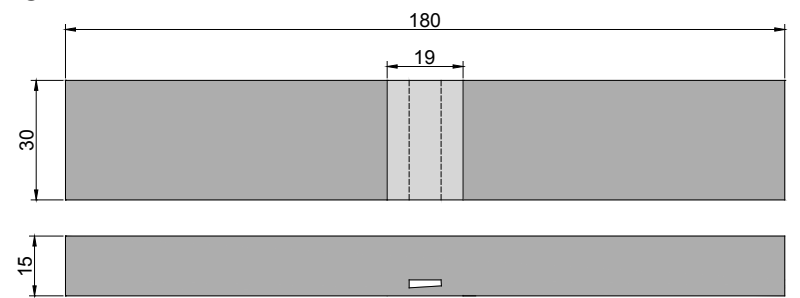

Figure 4. Geometry and dimensions of the fatigue specimens.

Fatigue tests were performed at room temperature on a servo-hydraulic Instron ${ }^{\circledR} 8502$ testing machine with a load cell of $100 \mathrm{kN}$. The run-out limit was set to $3 \times 10^{6}$ cycles. The loading regime was a load controlled, sinusoidal constant amplitude waveform with a $\min / \max$ stress ratio of $\mathrm{R}=0.1$, a frequency range of $[5,10] \mathrm{Hz}$ and a four-point bending set up.

The applied load, $\mathrm{P}$, was calculated by eq. 7 :
$P=\frac{\sigma b h^{2}}{3 w}$

where $\sigma$ is the maximum stress, $b$ is the specimen's width, $h$ is the specimen's thickness and $w$ is the smallest span distance $(30 \mathrm{~mm})$.

The specimens were tested with the processed surface under tensile stress and loads were applied out of the processed zone. Tests were carried out with the maximum stress levels ranging from $30-150 \mathrm{MPa}$, in order to determine the mean S-N curves.

After fatigue testing, fracture surfaces of failed specimens were observed in a scanning electron microscope (SEM).

\section{Numerical simulation}

\subsection{Finite element model}

Crack propagation on the nugget/thermo-mechanically affected zone (TMAZ) interface on the advancing side (AS) was analysed using the commercial FEM program ABAQUS. The specimen was modelled using a two-dimensional solid model, with a constant $30 \mathrm{~mm}$ depth. The channel and the nugget geometries were interpolated from a sample image, using the spline function to reconstruct the channel geometry (Figure 5). The $180 \times 30 \mathrm{~mm}$ section was subjected to four-point bending loads and boundary conditions, considering a $90 \mathrm{~mm}$ span.

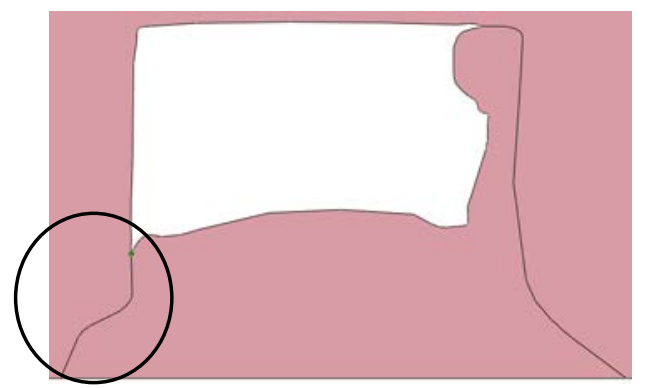

Figure 5. Channel nugget configuration. Crack propagation location along the interface.

\subsection{Fatigue failure simulation}

Different load amplitudes were applied for fatigue crack propagation simulation. The boundary conditions and loads were applied in four nodes, after verification that this simplification does not influence the results, when compared with the more complex simulation of the supports-specimen contact problem. By removing the supports and applying the boundary conditions in four nodes, the FEM analysis is linear and can be solved faster.

Both the base material and the channel nugget material where considered to be linear elastic, with properties shown in Table 2 and Table 5. Quadratic plane strain elements were used to model the geometry. A special meshing technique was used to define the crack tip and 
model stress singularity around the crack tip. Special wedge elements with collapse nodes, were used to determine the first contour integral, as show in Figure 6. A maximum of 50000 nodes were used. The energy release rate value, used for crack propagation calculation, was determined by the contour integral method. Crack propagation was allowed along the materials interface, using eq. 8. A constant crack increment $(\Delta \mathrm{a})$ was considered, and therefore the number of elapsed cycles between increments is calculated according with:

$N=\frac{\Delta a}{A \Delta G^{n}}$

The parameters for the Paris Law were estimated from the results obtained by Ilman et al. [17]. These authors. studied the crack propagation along a similar base material and friction stir welded material (Table 6), but not along the materials interface. Crack propagation was simulated with these two models and compared with the experimental results.

Table 5. Linear elastic properties for the base material and FSW material, used in FEM analysis [15].

\begin{tabular}{|l|l|l|}
\hline & $\begin{array}{l}\text { Young's } \\
\text { modulus (GPa) }\end{array}$ & $\begin{array}{l}\text { Poisson } \\
\text { rate }\end{array}$ \\
\hline Nugget & 69.8 & 0.3 \\
\hline
\end{tabular}

Table 6. Fatigue crack propagation parameters for the base material and FSW material, adapted from [17].

\begin{tabular}{|l|l|l|}
\hline & A (m/cycle) & n \\
\hline Base material & $1.365 \times 10^{-1}$ & 2.222 \\
\hline FSW & $1.116 \times 10^{-4}$ & 1.078 \\
\hline
\end{tabular}

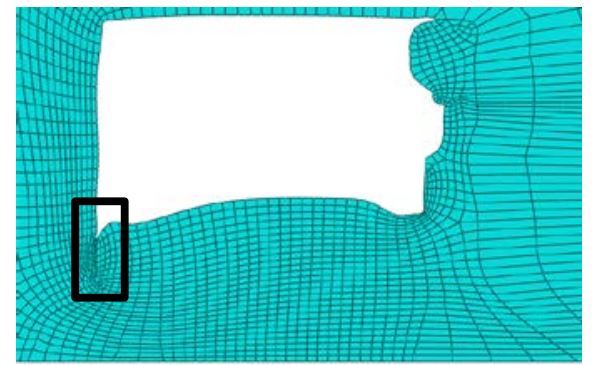

a)

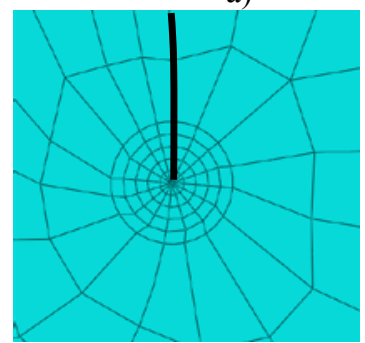

c)

Figure 6. Mesh and crack details. a) channel and nugget, b) crack and mesh along the crack, c) spider-web mesh for crack tip contour integral calculation.

\section{Results}

\subsection{Fatigue testing}

Table 7 presents the fatigue test results for the FSC specimens analysed.

Table 7. Fatigue test results.

\begin{tabular}{|l|l|l|l|l|}
\hline $\begin{array}{l}\text { Fatigue } \\
\text { Specimen } \\
\text { ID }\end{array}$ & $\begin{array}{l}\sigma_{\max } \\
\text { (MPa) }\end{array}$ & $\begin{array}{l}\text { Freq. } \\
\text { (Hz) }\end{array}$ & $\begin{array}{l}N_{f}, \\
\text { Cycles } \\
\text { to } \\
\text { failure }\end{array}$ & Obs. \\
\hline FSC_1 & 150 & 3 & 15259 & \\
\hline FSC_2 & 125 & 4 & 17094 & \\
\hline FSC_3 & 100 & 4 & 46165 & \\
\hline FSC_4 & 100 & 4 & 67293 & \\
\hline FSC_5 & 80 & 5 & 165572 & \\
\hline FSC_6 & 80 & 5 & 120796 & \\
\hline FSC_7 & 65 & 8 & 773309 & \\
\hline FSC_8 & 65 & 8 & 808165 & \\
\hline FSC_9 & 50 & 8 & 3000000 & Run-out \\
\hline
\end{tabular}

The S-N curves are presented in log-log scale. Each point represents an experimental test and the curves were obtained by fitting a linear regression eq. 8 , assuming $N_{f}$ as the dependent variable:

$$
\sigma_{\max }^{m} N_{f}=K_{0}
$$

where $\sigma_{\max }$ is the maximum stress, $N_{f}$ is the number of cycles to failure and $m$ and $K_{0}$ are empirical constants, namely the exponent and the coefficient of the S-N curve, respectively. The correlation lines fitted by eq. 8 represent the average regression curve with $50 \%$ of probability of failure. The regression lines are expressed by:

$$
\log \left(N_{f}\right)=\log \left(K_{0}\right)-m \log \left(\sigma_{\max }\right)
$$

In order to compare the fatigue test results, the S-N curves were described in terms of the fatigue strength at $2 \times 10^{6}$ cycles and the slope $m$, which is a recommended procedure to assess the fatigue strength of welded aluminium joints when the S-N curves derive directly from experimental data [18]. In addition, $K_{0}$ values and the standard error of the estimate (SEE) were also calculated for the regression line estimated. Results are presented in Table 8.

Table 8. Parameters of the mean curves.

\begin{tabular}{|l|c|c|c|c|}
\hline $\begin{array}{c}\text { Condition } \\
\text { ID }\end{array}$ & $m$ & $K_{0}$ & $\begin{array}{c}\text { SEE } \\
\text { (in } \\
\mathbf{l o g})\end{array}$ & $\begin{array}{c}\text { Fatigue } \\
\text { strength } \\
\text { at 2x10 } \\
\text { cycles } \\
\text { (MPa) }\end{array}$ \\
\hline BMa & 3.867 & $1.10 \times 10^{14}$ & 0.0651 & 100 \\
\hline FSC & 4.453 & $4.95 \times 10^{13}$ & 0.1717 & 46 \\
\hline
\end{tabular}

\subsection{Numerical simulations}

\footnotetext{
${ }^{\mathrm{a}}$ Specimens without channel.
} 
In order to study the fatigue crack propagation, a crack on the materials interface was allowed to propagate. The crack was simulated between the closest corner of the channel and the free surface. The initial crack size was considered to be $0.1 \mathrm{~mm}$ and the end condition occurred when the crack reached the free surface (until the channel loss of tightness). Figure 7 shows the evolution of the calculated energy release rate along vs the crack length. These values were used to calculate the number of elapsed fatigue cycles between a given crack increment. FSC specimen allow for a $3.3 \mathrm{~mm}$ crack length, as the crack changes direction along the interface between the base material and the nugget (Figure 5). As the crack opening mode changes from mode I to mode II, the energy release rate decreases slightly, slowing the crack propagation. Finally, the crack propagation rate will increase, as the crack length reaches the critical value of $21 \mathrm{~J} / \mathrm{mm}^{2}$.

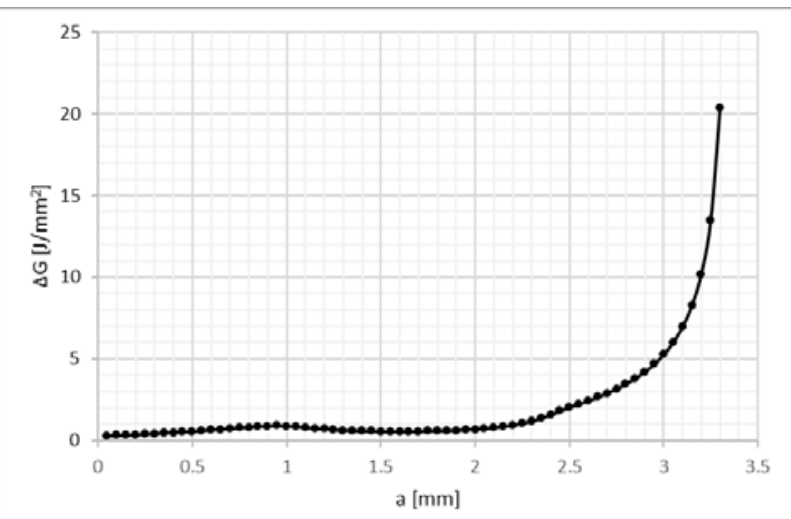

Figure 7. Energy release rate vs crack length, for a fatigue load with a maximum normal stress of $50 \mathrm{MPa}$.

Simulations of fatigue crack propagation were performed for different fatigue loads, and considering crack propagation properties of both, the base material and the FSW material. Table 9 summarizes the results. As concluded by Ilman et al. [17], the base material has a higher fatigue propagation resistance. Therefore, the obtained fatigue lives are always higher than the those obtained for the FSW material.

Table 9. Numerical fatigue simulation results.

\begin{tabular}{|c|c|c|}
\hline $\begin{array}{c}\sigma_{\max } \\
(\mathbf{M P a})\end{array}$ & $\begin{array}{c}\text { FSW - N } \\
\text { (Cycles) }\end{array}$ & $\begin{array}{c}\text { BM - N } \\
\text { (Cycles) }\end{array}$ \\
\hline 150 & 25728 & 40676 \\
\hline 125 & 38115 & 91454 \\
\hline 100 & 61662 & 246520 \\
\hline 80 & 99756 & 664510 \\
\hline 65 & 156080 & 1672000 \\
\hline 50 & 274780 & 5365000 \\
\hline
\end{tabular}

From the global analysis of the fatigue test results is possible to conclude that FSC specimens exhibit a very low fatigue strength comparing to the unprocessed base material, even though they have no defects.

As presented in Table 8 the scattering of the results is quite low which demonstrates that, on one hand it is possible to predict the fatigue behaviour of FSC components and, on the other hand, the technological process is stable and the channel is reproducible.

A thicker closing layer contributes to longer fatigue crack propagation periods, although it has been observed that for FSC specimens, the crack propagation period is residual when compared with the number of cycles required for crack initiation.

The fracture of all specimens took place in the nugget/TMAZ interface on the advancing side, as represented in Figure 8, where the dashed line symbolizes the crack propagation path. This interface is a region of stress intensity between very fine and coarse grains which is amplified by the channel corner geometry, as well as, presents the smallest resistant area.

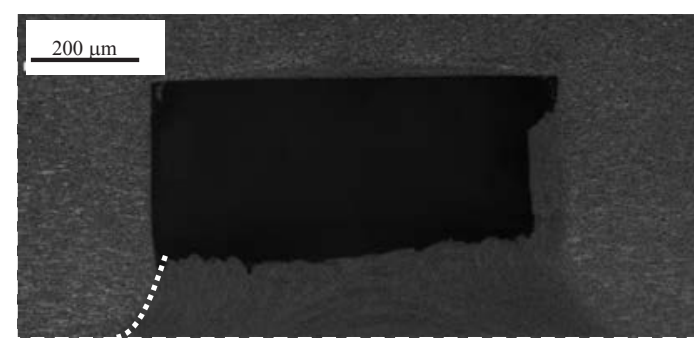

Figure 8. Crack path identification.

When considering both material models presented by Ilman et al. [17], one can see (Figure 9) that the fatigue lives predicted by the model presented herein and the base material properties, are higher than the experimental ones. This shows that this material model is not suited for the fatigue crack propagation of cracks on the interface between the base material and the FSW material. As mentioned this interface is considered to show a weak behavior. When considering the FSW material model, as seen in Figure 10, the predicted fatigue life represents $9 \%$ to $187 \%$ of the experimental life, as the fatigue load increases. Therefore the FSW material model is suitable for simulating fatigue crack propagation when the applied fatigue nominal stress has a maximum value of $100 \mathrm{MPa}$.

Within this loading range the model is in good agreement with the experimental results. The fatigue crack propagation life represents a maximum of $70 \%$ of the experimental life, for an $80 \mathrm{MPa}$ applied fatigue load. Nevertheless, this value is very high, as the experiments show that the crack initiation period represented the majority of the fatigue life.

\section{Discussion}




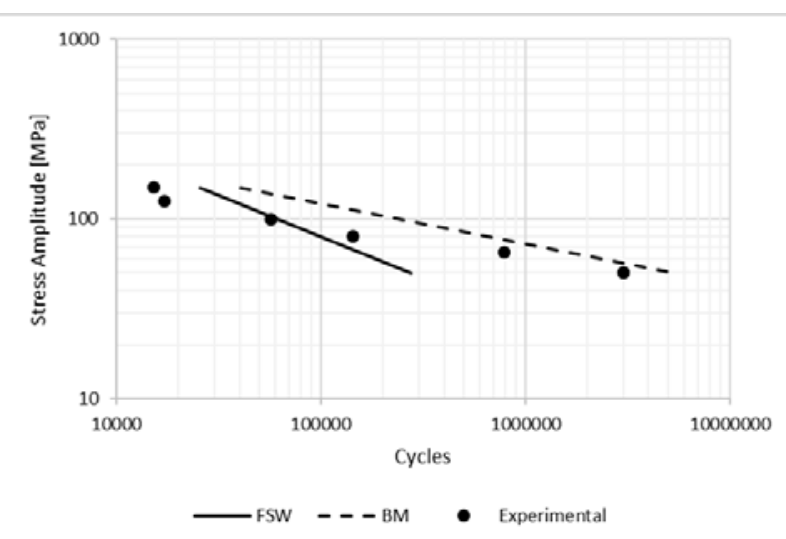

Figure 9. Fatigue life simulations for the FSC specimens.

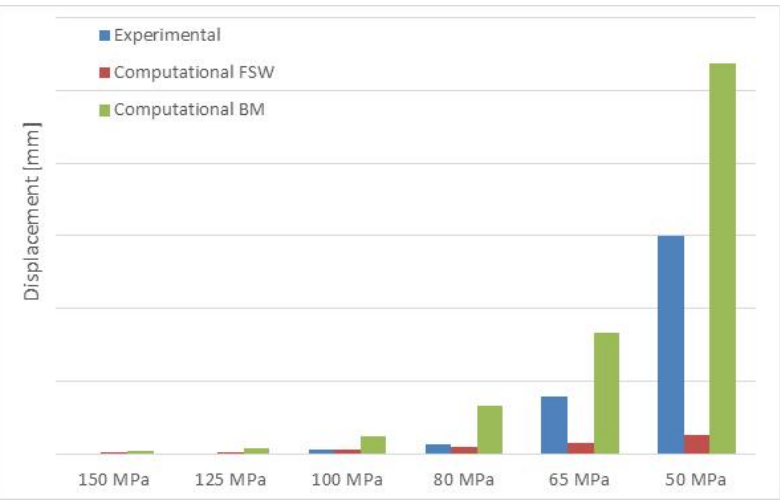

Figure 10. Comparison of experimental and computational values.

\section{Conclusions}

The global analysis of the fatigue test results have shown that FSC specimens exhibit a very low fatigue strength comparing to the unprocessed base material, even though they have no defects. A thicker closing layer contributes to longer fatigue crack propagation periods, although it has been observed that for FSC specimens, the crack propagation period is residual when compared with the number of cycles required for crack initiation. The fracture of all specimens took place in the nugget/TMAZ interface on the advancing side where a smallest resistant area is observed.

Based on the computational results one can conclude that the material model used is not suited for the fatigue crack propagation of cracks on the interface between the base material and the FSW material, when the applied fatigue loading is higher than $100 \mathrm{MPa}$. The fatigue crack propagation life obtained in the computational simulations represents a maximum of $70 \%$ of the experimental life, for an applied fatigue loads of 80 $\mathrm{MPa}$. Nevertheless, this value is very high, as the experiments show that the crack initiation period represented the majority of the fatigue life. Future work should be done to quantify the fatigue crack initiation and propagation periods, in order to better choose the correct material model for the fatigue crack propagation simulations.

\section{Acknowledgements}

The authors would like to acknowledge the Portuguese Foundation for the Science and Technology (FCT) for its financial support through the $\mathrm{PhD}$ scholarship FCT SFRH/BD/62963/2009.

\section{References}

1. Thomas, W.M., Friction stir welding and related friction process characteristics, 7th International Conference Joints in Aluminium, Inalco, TWI, Cambridge, 1998.

2. Thomas, W.M., Sylva, G., Developments of friction stir welding, ASM Materials Solutions 2003, Conference and Exposition, Pittsburgh, Pennsylvania, USA, 2003.

3. Thomas, W.M., Nicholas, D.E., Staines, D.G., Tubby, P.J., Gittos, M.F., FSW Process Variants and Mechanical Properties, Doc IIW-1664-04, Welding in the World 49 (3/4), pp 4-11, 2005.

4. Thomas, W.M., Norris, I.M., Staines, D.G., Clarke, P.J., Horrex, N.L., Friction stir welding - variants and process techniques, The First International Conference Joining of Aluminium Structures, Moscow, Russian, 2007.

5. Mishra, R.S., Integral channels in metal components and fabrication thereof, Patent no. US 6923362 B2, August 2, 2005.

6. Fajdiga, G., Sraml, M., Fatigue crack initiation and propagation under cyclic contact loading, Engineering Fracture Mechanics, 76 (20069), pp. 1320-1335.

7. Cheng, W., Cheng, H.S., Mura, T., Keer, L.M:, Micromechanics modelling of crack initiation under contact fatigue, ASME Journal Tribology, 1994, 116, pp. 2-8.

8. Zhang, H., Yang, J., Hu, J., Li, X., Li, M., \& Wang, C. (2018). An experimental and simulation study of interface crack on zinc coating/304 stainless steel. Construction and Building Materials, 161, 112-123. https://doi.org/10.1016/j.conbuildmat.2017.11.022

9. Kakiuchi, T., Uematsu, Y., \& Suzuki, K. (2016). Evaluation of fatigue crack propagation in dissimilar $\mathrm{Al} /$ steel friction stir welds. Procedia Structural Integrity, 2, 1007-1014. https://doi.org/10.1016/j.prostr.2016.06.129

10. Shah, S. G., Ray, S., \& Chandra Kishen, J. M. (2014). Fatigue crack propagation at concreteconcrete bi-material interfaces. International Journal of Fatigue, 63, 118-126. https://doi.org/10.1016/j.ijfatigue.2014.01.015

11. Suo, Z. G., \& Hutchinson, J. W. (1990). Interface Crack Between 2 Elastic Layers. International Journal of Fracture, 43(5), 1-18. https://doi.org/Doi $\underline{10.1007 / \mathrm{Bf00018123}}$ 
12. Dundurs J. Edge bonded dissimilar orthogonal elastic wedges under normal and shear loading. J Appl Mech 1969;36:650.

13. Rice JR, Sih GC. Plane Problems of Cracks in Dissimilar Media. ASME. J. Appl. Mech. 1965;32(2):418-423. doi:10.1115/1.3625816.

14.Xuan, F. Z., Tu, S. T., \& Wang, Z. (2005). A modification of ASTM E $1457 \mathrm{C}^{*}$ estimation equation for compact tension specimen with a mismatched cross-weld. Engineering Fracture Mechanics, 72(17), 2602-2614. https://doi.org/10.1016/j.engfracmech.2005.05.002

15. Vidal, C., Infante, V., Vilaça, P., Fatigue behaviour at elevated temperature of friction stir channelling solid plates of AA5083-H111 aluminium alloy, International Journal of Fatigue, Volume 62, May 2014, pp. 85-92, https://doi.org/10.1016/j.ijfatigue.2013.10.012

16. Vidal, C., Infante, V., Vilaça, P., Assessment of Performance Parameters for Friction Stir Channelling, Proceedings of the IIW 2011 International Conference on Global Trends in Joining, Cutting and Surfacing Technology, Chennai, India, 21-22 July, 2011, ISBN 978-81-8487-152-4, paper IC_99.

17. M.N.Ilman, W. (2013). Fatigue crack growth behaviour of shot peened 5083 aluminium alloy friction stir welds. International Conference on Joining Materials, (May 2013).

18. Maddox, S.J., Review of fatigue assessment procedures for welded aluminium structures, International Journal of Fatigue 25, pp 1359 1378, 2003. 